\title{
Principios y calidad humana en la educación médica: perspectiva de un estudiante de medicina humana
}

\author{
Carlos J. Toro-Huamanchumo
}

Recientemente se publicó en la revista un interesante artículo de Soler-González et al [1] acerca de las competencias del estudiante de medicina humana para respetar la idiosincrasia y etnia del paciente. En el estudio se describe y hace una sucinta reflexión acerca de las diferentes percepciones y actitudes de los estudiantes en el momento de la anamnesis, diagnóstico y tratamiento según la etnia del paciente, lo cual es un claro indicador de que la existencia de las inequidades en salud tienen como base una deficiente formación en principios y calidad humana durante la etapa de pregrado.

La inequidad en salud se define como el conjunto de diferencias innecesarias, injustas y evitables que puedan existir en el sector de la salud [2]. Estas diferencias constituyen un problema de ámbito mundial y se centran principalmente en los países de Latinoamérica y el Caribe $[2,3]$. Perú constituye un ejemplo claro de país donde aún existen brechas en distintos sectores sociales, lo cual genera un ambiente de injusticia, desigualdad y exclusión en un sistema de salud que se encuentra segmentado y fragmentado [4].

La reducción de las disparidades en la salud, producto de la extrapolación de prejuicios y estereotipos sociales al campo profesional, depende de la capacidad del médico para entender efectivamente, comunicar y saber tratar los diversos grupos de pacientes $[1,5]$. Por ello, la etapa de pregrado resulta una oportunidad idónea para cultivar habilidades y actitudes basadas en el respeto y la ecuanimidad. De este modo, más adelante se asegura la cosecha de médicos que respeten la autonomía, creencia y cultura del paciente [1].

Las facultades de medicina deben priorizar la formación de profesionales con una adecuada capacitación cognitiva, moral y cultural, que puedan aportar positivamente a la sociedad [6,7]. Cursos como bioética y filosofía son importantes para formar el carácter del estudiante y educarlo moral- mente. Los docentes que dicten estos cursos no deben imponer un tipo de pensamiento único, sino servir de guías para que el alumno construya su propio pensamiento crítico basado en principios y valores. El ámbito hospitalario también tiene un rol importante en la formación ética y humanística, pues representa el complemento práctico de la teoría dada en la universidad, y en él se debe promover la enseñanza de una atención médica que respete el derecho del paciente [7].

Por otra parte, las sociedades científicas de estudiantes de medicina refuerzan lo mencionado anteriormente. En ellas no sólo se promueve el amor hacia la investigación, sino también a la labor social en beneficio de las comunidades vulnerables. Además, mediante la investigación en salud pública se pueden identificar las categorías -aparte de la etniaque dan forma a la salud de las poblaciones [5].

Finalmente, es importante considerar que para asegurar una educación médica excelente y una calidad profesional mañana, hay que asegurar una calidad humana hoy.

Bibliografía

1. Soler-González J, Rodríguez-Rosich A, Marsal-Mora JR, en representación del grupo AP-UDL. Competencia del estudiante de medicina para respetar las creencias y la cultura del paciente. FEM 2014; 17: 69-74.

2. Cardona D, Acosta LD, Bertone CL. Inequidades en salud entre países de Latinoamérica y el Caribe (2005-2010). Gac Sanit 2013; 27: 292-7.

3. Marmot M, Allen J, Bell R, Goldblatt P. Building of the global movement for health equity: from Santiago to Rio and beyond. Lancet 2012; 379: 181-8.

4. Maguiña C, Galán-Rodas E. Situación de la salud en el Perú: la agenda pendiente. Rev Peru Med Exp Salud Publica 2011; 28: 569-70.

5. Powell-Sears K. Improving cultural competence education: the utility of an intersectional framework. Med Educ 2012; 46: 545-51.

6. Martins-Silva J. Educação médica e profissionalismo. Acta Médica Port 2013; 26: 420-7.

7. Perales-Cabrera A. Ética y humanismo en la formación médica. Acta Bioethica 2008; 14: 30-8.
Sociedad Científica de Estudiantes de Medicina Veritas. Facultad de Medicina Humana. Universidad de San Martín de Porres. Chiclayo, Perú.

Correspondencia:

Est. Carlos J. Toro Huamanchumo. Sociedad Científica de Estudiantes de Medicina Veritas (SCIEMVE). El Muelle Ltes. 5, 6 y 7. Las Delicias. Chiclayo 14001. Lambayeque, Perú.

E-mail:

toro2993@hotmail.com

(c) 2015 FEM 